\title{
Sinterização de porcelanatos com fluxo viscoso: Uma revisão
}

\author{
Chiara Zanelli', Matteo Ardit², Sonia Conte ${ }^{1}$, Roberto Soldati', Giuseppe Cruciani ${ }^{2}$, Michele Dondi ${ }^{1}$ \\ ${ }^{I}$ CNR-ISTEC, Instituto de Ciência e Tecnologia de Materiais Cerâmicos, Faenza, Itália. \\ ${ }^{2}$ Departamento de Ciências Físicas e da Terra, Universidade de Ferrara, Itália. \\ *e-mail: michele.dondi@istec.cnr.it
}

\begin{abstract}
Resumo:
Os porcelanatos, como todos materiais porcelânicos, são sinterizados por vitrificação sob fluxo viscoso de uma fase líquida abundante formada em altas temperaturas. Este processo deve ser mantido sob controle rigoroso para se obter as propriedades desejadas dos produtos finais e para prevenir os defeitos induzidos pela piroplasticidade. Principalmente para placas de grandes formatos, onde a produção prima por uma densidade uniforme das placas e por mínima deformação à alta temperatura. Este trabalho faz uma revisão crítica do estado da arte da sinterização de porcelanatos e dos desafios para o desenvolvimento de placas de grande formato. Será discutido o nível de conhecimento nos distintos fenômenos envolvidos na sinterização com fluxo viscoso, diferenciando-se entre o que já é conhecido, o que é reconhecido, mas ainda necessita completo entendimento, e o que pode aparecer de uma compreensão mais profunda. Fatos conhecidos: a evolução da microestrutura e da composição de fases durante a queima é bem conhecida, e os modelos fenomenológicos têm sido desenvolvidos para a densificação por fluxo viscoso de porcelanatos. Os efeitos combinados da viscosidade da fase líquida e do teor de sólidos sobre a piroplasticidade foram revelados. É conhecida a forma como a razão $\mathrm{Na} / \mathrm{K}$ e outras relações químicas da composição dos porcelanatos afeta a cinética de sinterização. Também foi reconhecido o papel da cristalização/dissolução da mulita e da transição do quartzo alfa para beta sobre as propriedades tecnológicas. Os fatos não tão conhecidos: alterações dinâmicas na composição e nas propriedades físicas da fase líquida durante a queima são previstas, mas a vitrificação e a sinterização reativa ainda não foram modeladas. Há incertezas em relação ao efeito real dos cristais suspensos no meio líquido sobre a cinética de sinterização e sobre a piroplasticidade em função de sua forma e distribuição de tamanho variáveis. São obscuros o efeito da porosidade inicial sobre o grau e cinética de densificação, a homogeneidade e a miscibilidade das fases líquidas, a solubilidade de gás na fase líquida na maior temperatura de queima (e seu papel na porosidade fechada e no inchamento), a dependência da razão $\mathrm{Fe}^{2+} / \mathrm{Fe}^{3+}$ sobre a temperatura e sua consequência sobre a viscosidade da fase líquida. O que é desconhecido: a modelagem das curvas de densificação e a predição das propriedades físicas dos porcelanatos, por cálculos computacionais, pode prefigurar o papel de outras variáveis ainda não consideradas. A extensão de tais esforços de modelagem da escala laboratorial para a industrial pode revelar a ocorrência de efeitos de escala, principalmente para o caso de placas grandes e para distintas técnicas de formação. Uma melhor compreensão de aspectos cinéticos resultantes da convolução de vários fatores pode revelar resultados inesperados, tais como efeitos de memória das matérias-primas ou características microestruturais.
\end{abstract}

Palavras-chave: Sinterização; Fase líquida viscosa; Microestrutura; Porcelanato.

\section{Introdução:}

Os porcelanatos são fabricados por queima rápida, tipicamente a uma temperatura máxima entre $1200-1230{ }^{\circ} \mathrm{C}$ e ciclos (frio a frio) de 50-70 minutos. Como nos materiais porcelânicos, a sinterização ocorre por vitrificação com consequente fluxo viscoso de uma abundante fase líquida formada a elevadas temperaturas $[1,2]$. Este processo implica uma rápida taxa de densificação (5-8\% de retração linear em 8-10 min) que deve ser mantida sob controle rigoroso para se obter as propriedades desejadas nos produtos finais $[3,4]$. Principalmente, a absorção de água deve ser mantida abaixo de $0,5 \%$ e os defeitos relacionados à piroplasticidade devem ser prevenidos [5-7].

A preocupação com o controle da queima é ainda mais evidente para o caso das placas de grande formato, p.ex., $360 \times 120 \mathrm{~cm}$, cuja produção requer densificação uniforme e deformações mínimas à alta temperatura $[8,9]$. A partir deste ponto de vista, este trabalho objetiva fazer uma revisão crítica do estado da arte da sinterização de porcelanatos, com ênfase nos desafios para o desenvolvimento de placas de grande formato. Esta revisão segue o nível de conhecimento obtido na literatura para distintos fenômenos envolvidos na sinterização com fluxo viscoso, inicialmente em função de conhecimentos prévios (os fatos conhecidos). Em seguida, a discussão difere o que já é reconhecido, mas ainda necessita ser completamente entendido (os fatos não tão conhecidos) $\mathrm{e}$ o que pode surgir pela maior compreensão do que ocorre na sinterização com fluxo viscoso (o que é desconhecido). 


\subsection{Os fatos conhecidos:}

Os mecanismos que governam a sinterização com fluxo viscoso são conhecidos (Fig.1). Após o estágio inicial, onde a sinterização é essencialmente governada pela difusão superficial com redução progressiva da área superficial específica, ocorre a formação de uma grande quantidade de líquido em um curto intervalo de temperatura $\left(1000-1100^{\circ} \mathrm{C}\right)$. Isto dá origem a um fluxo viscoso controlado pela viscosidade e tensão superficial da fase líquida, que por sua vez é influenciada pela solubilidade dos cristais no líquido. Tal processo resulta em uma rápida densificação, o que caracteriza o estágio intermediário. A taxa de sinterização reduz gradualmente, enquanto a máxima densidade possível é obtida no estágio final, onde a viscosidade e solubilidade do gás no líquido controlam o crescimento dos poros, podendo ocorrer o fenômeno de inchamento [10-12].

As curvas de densificação para porcelanato são conhecidas em detalhes, tanto em condições industriais quanto laboratoriais, e foram propostos modelos fenomenológicos [13-16]. Este conhecimento fundamentou a primeira tentativa de se fazer uma predição computacional da retração de queima em porcelanatos [17] por analogia com produtos sanitários [18-19].

A evolução combinada da microestrutura e da composição de fases durante a queima tem sido muito investigada nos porcelanatos $[2,20-31]$ e nas porcelanas vitrificadas [7,30-31]. Isto permite um quadro geral das transformações de fases que ocorrem nos componentes minerais dos corpos porcelânicos (Fig.2):

Os argilominerais se decompõem e transformam-se em fases amorfas [25,27];

Fusão de feldspatos, envolvendo os remanescentes amorfos das argilas e as fases de sílica [2,27,30];

Formação de novos compostos, principalmente mulita e fases transientes [20,25,31] que podem estar presentes nos porcelanatos (espinélio de $\mathrm{Al}-\mathrm{Si}$, anortoclásio, sanidina);

Fases refratárias persistentes $[25,30,31]$ mesmo que parcialmente dissolvidas no líquido (quartzo, mulita, zirconita, rutilo).

Estas transformações de fases implicam que ocorre uma evolução microestrutural paralela ao aumento de temperatura, a partir do início da junção dos grãos de feldspato e quartzo dispersos, que vão formar uma matriz de grãos finos que acomoda os argilominerais e a porosidade (Fig.3a). A vitrificação causa as principais alterações microestruturais pela formação, a partir de $1000-1100{ }^{\circ} \mathrm{C}$, de regiões vítreas (Fig.3b). Este líquido molha os grãos cristalinos e sua permeação através dos grãos resulta na densificação no intervalo entre $1100-1200^{\circ} \mathrm{C}$ (Fig.3c). Além da vitrificação e do fluxo viscoso ocorrem reações sólido-líquido, principalmente a fusão parcial do quartzo e dissolução/cristalização da mulita, com eventual crescimento de cristais alongados de mulita secundária (Fig.3d). A microestrutura final consiste em uma fase vítrea com cristais embebidos [21-23,28,29]. Tais características podem ser comparadas àquelas observadas em porcelana normal e vitrificada [7,30-33].
As transformações térmicas podem resultar em deformações em alta temperatura, comumente chamadas de piroplasticidade $[5,6]$. É geralmente reconhecido que a viscosidade da fase líquida e o teor de sólidos (quantidade, tamanho e forma dos cristais) governam o comportamento piroplástico [34-40]. Por outro lado, sabe-se que a razão $\mathrm{Na}_{2} \mathrm{O} / \mathrm{K}_{2} \mathrm{O}$ e outros fatores químicos da composição dos porcelanatos afetam a cinética de sinterização $[34,37,38,41-46]$ como ocorre nos materiais porcelânicos [47,51]. Este controle é exercido em função da quantidade e propriedades físicas da fase vítrea, que varia de acordo com a composição química do líquido fundido $[2,52]$.

O papel da mulita no desempenho técnico dos porcelanatos já foi muito estudado [25,47,53-58]. Tanto a cinética da formação da mulita a partir de metacaulim $[47,53,56-58]$ e o desenvolvimento da mulita secundária $[20,27,54,59]$ são particularmente preocupantes.

O papel da transformação do quartzo $\beta \leftrightarrow \alpha$ é amplamente reconhecido, não apenas para possíveis rupturas durante o resfriamento [60-64], mas também pelo seu efeito nas propriedades elásticas e tensões residuais [61,64-66].

\subsection{Os fatos não tão conhecidos}

As transformações microestruturais e de fases, como mostradas nas Figs. 2 e 3, implicam em alterações dinâmicas na construção de fases e composição química do

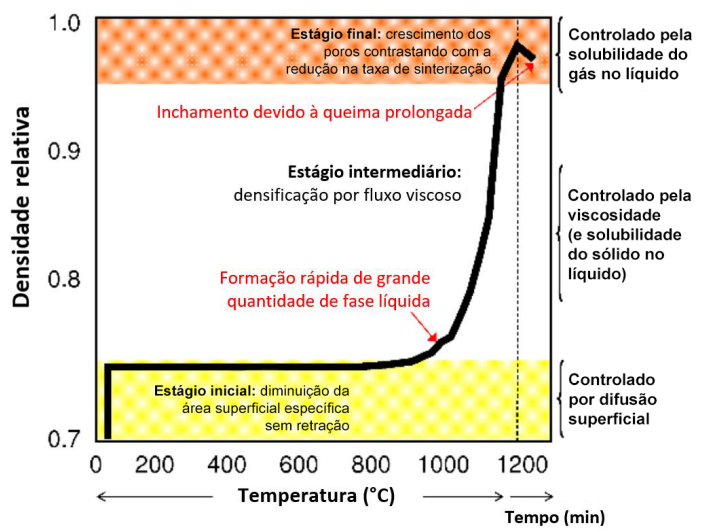

Figura 1. Estágios de sinterização e mecanismos ativos durante a queima de porcelanato

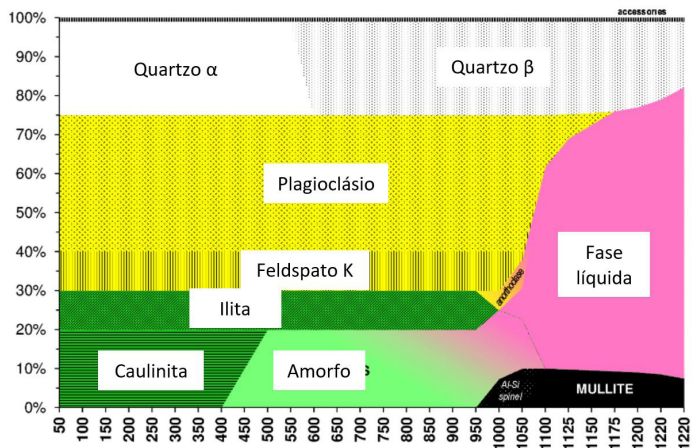

Figura 2. Evolução de fases com a temperatura de queima de um porcelanato típico 
fundido. Consequentemente, as propriedades físicas da fase líquida, que dependem de suas características físicas, são alteradas continuamente durante a queima. Esta evolução é conhecida apenas aproximadamente [1,2], apesar de seu papel fundamental na vitrificação e sinterização, e ainda não foi modelada.

Há incertezas em relação ao efeito real dos cristais imersos no fundido sobre a cinética de sinterização e sobre a piroplasticidade. Apesar de haver evidências do papel determinante da existência de um teor de sólidos variável, ainda existem opiniões contrastantes sobre o efeito da forma e tamanho dos cristais nas deformações de queima $[5,7,21,35,39,40]$. O caso da mulita é emblemático. Seu efeito sobre as propriedades mecânicas dos porcelanatos ainda é debatido. Foi reportada uma correlação direta entre o módulo de ruptura e a tenacidade à fratura com o teor de mulita [67-69], relacionada a mecanismos de tenacificação análogos àqueles conhecidos para a porcelana [70]. Entretanto, o percentual de mulita presente no porcelanato é muito menor que nas porcelanas [2,70], e em muitos casos surge uma relação adversa com as propriedades mecânicas [24,71,72]. Este comportamento variável depende das complexas características microestruturais da mulita, incluindo uma possível ocorrência de cristais secundários alongados, e de sua cristalização/dissolução durante o caminho de vitrificação [73].
$\mathrm{Na}$ tentativa de baixar a temperatura de queima dos porcelanatos, cresceu o interesse por fluxos fortes e não convencionais, p.ex., contendo grandes quantidades de óxidos alcalinos-terrosos ou compostos de boro [34,42,43,46]. Nestes corpos foi descoberto um comportamento em queima peculiar [44], ainda não completamente entendido. Em vez das tradicionais curvas de queima, que convergem para uma absorção de água mínima que combina com a máxima retração e máxima densidade aparente (Fig.4a), pode ser que a absorção de água mínima seja obtida somente após a inversão da retração e da densidade aparente (Fig.4b).

$\mathrm{O}$ efeito da porosidade inicial sobre o grau de densificação ainda é indefinido. Foi observado que quanto maior for o volume de poros em placas cruas, maior é a cinética de sinterização [12,59]. Embora um menor valor de densidade aparente para as placas cruas leve a porcelanatos menos densos, após o mesmo ciclo de queima, a diferença em relação à porosidade inicial é recuperada durante a sinterização, e os valores finais de densidade aparente apresentam uma pequena diferença. A influência da porosidade inicial é reconhecida na sinterização de vidros [74], mas a sua relevância nos porcelanatos ainda deve ser provada.

Outro tema questionável é a homogeneidade e miscibilidade das fases líquidas formadas nos porcelanatos. Geralmente pensa-se que o tempo disponível em uma queima rápida é insuficiente para permitir que um

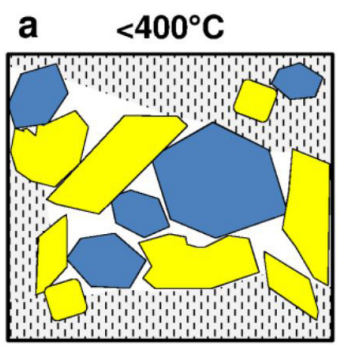

Quartzo

Feldspato

Matriz argilosa b $\quad \sim 1100^{\circ} \mathrm{C}$

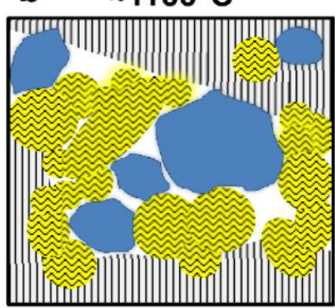

Mulita c/ matriz amorfa Líquido feldspático

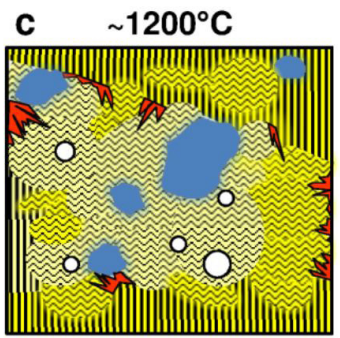

d $>1250^{\circ} \mathrm{C}$

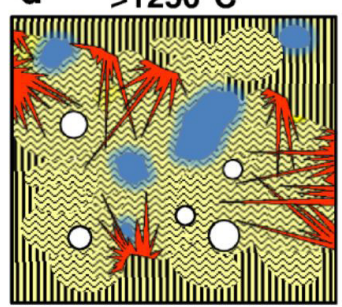

Mulita secundária

Poros

\section{Líquido quartzo-feldspático}

Figura 3. Evolução microestrutural de materiais porcelânicos durante a queima
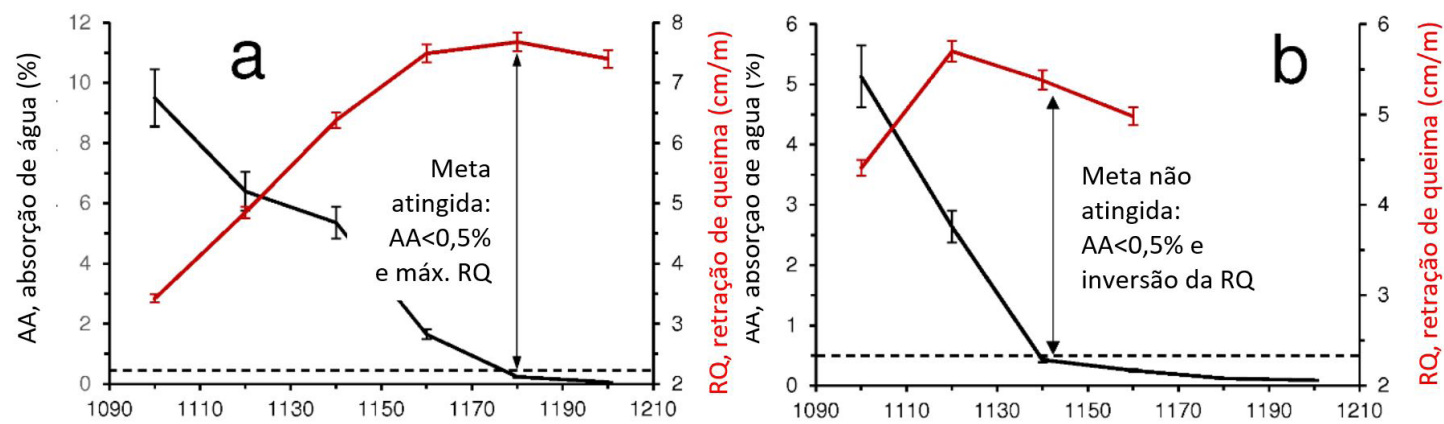

Figura 4. Curvas de sinterização para (a) um porcelanato típico e (b) uma composição modificada contendo $2,5 \%$ de um forte fluxo (ulexita) [44] 
fundido homogêneo seja obtido, pois muitas pesquisas sobre microestruturas destacam a formação de gradientes de composição na fase vítrea de porcelanas queimados lentamente $[32,33,73]$. Entretanto, a difusão de álcalis em silicatos fundidos é muito rápida, e evidências experimentais mostram que esta pode ocorrer em um intervalo de tempo de minutos [75].

Outra questão pendente refere-se ao papel da solubilidade dos gases em fases líquidas a altas temperaturas, quando a pressão de sinterização diminui e a porosidade fechada pode aumentar, resultando em inchamento para uma queima prolongada. O vapor de água pode aumentar a cinética de sinterização em porcelanas [76]. Além disto, foi previsto que a solubilidade do gás de combustão pode mudar com as variações na viscosidade e na composição do fundido [77], mas não há evidência experimental para porcelanatos.

Por outro lado, acredita-se que o oxigênio liberado devido à redução do ferro (p.ex., na quebra da hematita: $\mathrm{Fe}_{2} \mathrm{O}_{3} \rightarrow 2 \mathrm{FeO}+1 / 2 \mathrm{O}_{2}$ ) possa contribuir para o aumento dos poros no estágio final da sinterização [78]. Além disto, a ocorrência de $\mathrm{Fe}^{2+}$ pode diminuir significativamente a viscosidade do fundido [79]. Entretanto, não há dados disponíveis sobre a dependência da razão $\mathrm{Fe}^{2+} / \mathrm{Fe}^{3+}$ em relação à temperatura de queima.

\subsection{Os fatos desconhecidos:}

A predição do comportamento de queima das placas cerâmicas é desafiador, particularmente para os porcelanatos, devido a vários fenômenos que ocorrem em uma escala múltipla: da mobilidade atômica (p.ex., difusão iônica através do fundido) ao rearranjo microestrutural (consequências das transformações de fase e vitrificação) e à retração e deformações em relação ao produto como um todo.

A necessidade de cálculos computacionais para o modelamento do comportamento de queima demandará esforços adicionais para melhorar a compreensão do fenômeno implicado na sinterização de porcelanatos. Este processo pode mostrar o papel de variáveis adicionais, ainda não consideradas, ou a interdependência dos fatores.

A ampliação de tais esforços de modelamento, do laboratório para a produção industrial, revelará a ocorrência de efeitos de escala, principalmente no caso de placas grandes, e uma possível dependência no processamento tecnológico (p.ex., diferentes técnicas de formação).

Uma melhor compreensão dos aspectos cinéticos, resultado da convolução de vários fatores, pode revelar resultados inesperados, como efeitos de memória das matérias-primas ou características microestruturais.

\section{AGRADECIMENTOS}

Os autores agradecem à região da Emília-Romagna pelo suporte financeiro, por meio do projeto 'IPERCER: inovação de processo para a cadeia de valor de placas cerâmicas sustentáveis', POR FESR 2014-2020 (eixo 1, ação 1.2.2).

\section{REFERÊNCIAS}

[1] Carty, W.M., 2002. Observations on the glass phase composition in porcelains. Ceramic Engineering and Science Proceedings, 23, 79-94.

[2] Zanelli, C., Raimondo, M., Guarini, G., Dondi, M., 2011. The vitreous phase of porcelain stoneware: composition, evolution during sintering and physical properties. Journal of Non-Crystalline Solids, 357, 3251-3260.

[3] Sánchez, E., García-Ten, J., Sanz, V., Moreno, A., 2010. Porcelain tile: Almost 30 years of steady scientific-technological evolution. Ceramics International, 36, 831-845.

[4] Romero, M., Pérez, J.M., 2015. Relation between the microstructure and technological properties of porcelain stoneware-A review. Materiales de Construcción, 65, e065.

[5] Buchtel, A.M., Carty, W.M., Noirot, M.D., 2009. Pyroplastic deformation revisited. Whitewares and Materials: Ceramic Engineering and Science Proceedings, 25, 25-42.

[6] Bresciani, A., Spinelli, B., 2012. Porcelain tile pyroplastic deformation during firing and post-firing variations of planarity. CFI Ceramic Forum International, 89, E41-E45.

[7] Porte, F., Brydson, R., Rand, B., Riley, F. L., 2004. Creep viscosity of vitreous china. Journal of the American Ceramic Society, 87, 923-928.

[8] Zanelli, C., Raimondo, M., Guarini, G., Marani, F., Fossa, L., Dondi, M., 2010. Porcelain stoneware large slabs processing and technological properties. Proc. XI Congreso Mundial de la Calidad del Azulejo y del Pavimento, QUALICER, Castellon.

[9] Bresciani, A., Ricci, C., 2014. Continuous compaction of ceramic slabs with integrated fastening system. Proc. XIII Congreso Mundial de la Calidad del Azulejo y del Pavimento, QUALICER, Castellon.

[10]F. Cambier, A. Leriche, 1996. Vitrification. In: R.W. Cahn, P. Hanson, E.J. Kramer (Eds.), Processing of Ceramics Part II, vol 17B, VCH Basel, pp. 124-144.

[11]Zanelli, C., Raimondo, M., Dondi, M., Guarini, G., Tenorio, P., 2004. Sintering mechanisms of porcelain stoneware tiles. Proceedings Qualicer 2004, 247-259.

[12] Salem, S., Salem, A., 2013. Mechanisms of momentum transport in viscous flow sintering. INTECH Open Access Publisher.

[13] Orts, M. J., Amorós, J. L., Escardino, A., Gozalbo, A., Feliu, C., 1993. Kinetic model for the isothermal sintering of low porosity floor tiles. Applied Clay Science, 8, 231-245.

[14] Salem, A., Jazayeri, S. H., Rastelli, E., Timellini, G., 2010. Kinetic model for isothermal sintering of porcelain stoneware body in presence of nepheline syenite. Thermochimica Acta, 503, 1-7.

[15] Salem, S., Salem, A., 2014. Shrinkage prediction during non-isothermal sintering in the presence liquid phase: New kinetic model, Part I. Thermochimica Acta, 575, 322-330.

[16] Salem, S., Salem, A., (2014).Effects of technical factors on dimensional stability of porcelain stoneware body during sintering process: Isothermal kinetic study, Part II.Thermochimica Acta, 598, 82-90.

[17] Pavlovic, A., Fragassa, C. 2016. Modelling the viscoelasticity of ceramic tiles by finite element. AIP Conference Proc., 1736, art. no. 4949749.

[18] Bene, P., Bardaro, D., 2014. Numerical-experimental method to study the viscous behaviour of ceramic materials. Journal of the European Ceramic Society, 34, 2617-2622. 
[19]De Miranda, S., Patruno, L., Ricci, M., Saponelli, R., Ubertini, F., 2015. Ceramic sanitary wares: Prediction of the deformed shape after the production process. Journal of Materials Processing Technology, 215, 309-319.

[20] Sanchez, E., Orts, M. J., Garcia-Ten, J., Cantavella, V., 2001. Porcelain tile composition effect on phase formation and end products. American Ceramic Society Bulletin, 80, 43-49.

[21]Leonelli, C., Bondioli, F., Veronesi, P., Romagnoli, M., Manfredini, T., Pellacani, G. C., Cannillo, V., 2001. Enhancing the mechanical properties of porcelain stoneware tiles: a microstructural approach. Journal of the European Ceramic Society, 21, 785-793.

[22] Souza, G. P., Rambaldi, E., Tucci, A., Esposito, L., Lee, W. E., 2004. Microstructural variation in porcelain stoneware as a function of flux system. Journal of the American Ceramic Society, 87, 1959-1966.

[23] Sanchez, E., Ibanez, M. J., García-Ten, J., Quereda, M. F., Hutchings, I. M., Xu, Y. M., 2006. Porcelain tile microstructure: implications for polished tile properties. Journal of the European Ceramic Society, 26, 2533-2540.

[24] Carbajal, L., Rubio-Marcos, F., Bengochea, M. A., Fernandez, J. F., 2007. Properties related phase evolution in porcelain ceramics. Journal of the European Ceramic Society, 27, 4065-4069.

[25] Gualtieri, A. F., 2007. Thermal Behavior of the Raw Materials Forming Porcelain Stoneware Mixtures by Combined Optical and In Situ X-Ray Dilatometry. Journal of the American Ceramic Society, 90, 1222-1231.

[26] Martín-Márquez, J., Rincón, J. M., Romero, M., 2008. Effect of firing temperature on sintering of porcelain stoneware tiles. Ceramics International, 34, 1867-1873.

[27] Martín-Márquez, J., De la Torre, A. G., Aranda, M. A., Rincón, J. M., Romero, M., 2009. Evolution with temperature of crystalline and amorphous phases in porcelain stoneware. Journal of the American Ceramic Society, 92, 229-234.

[28] De Noni, A., Hotza, D., Soler, V. C., Vilches, E. S., 2010. Influence of composition on mechanical behaviour of porcelain tile. Part I: Microstructural characterization and developed phases after firing. Materials Science and Engineering, A527, 1730-1735.

[29]Pérez, J. M., Romero, M., 2014. Microstructure and technological properties of porcelain stoneware tiles moulded at different pressures and thicknesses. Ceramics International, 40, 1365-1377.

[30]Pagani, A., Francescon, F., Pavese, A., Diella, V., 2010. Sanitary-ware vitreous body characterization method by optical microscopy, elemental maps, image processing and X-ray powder diffraction. Journal of the European Ceramic Society, 30, 1267-1275.

[31]Bernasconi, A., Diella, V., Pagani, A., Pavese, A., Francescon, F., Young, K., J. Stuart Tunnicliffe, L., 2011. The role of firing temperature, firing time and quartz grain size on phase-formation, thermal dilatation and water absorption in sanitary-ware vitreous bodies. Journal of the European Ceramic Society, 31, 1353-1360.

[32] Iqbal, Y., Lee, W. E., 1999. Fired porcelain microstructures revisited. Journal of the American Ceramic Society, 82, 3584-3590.

[33] Iqbal, Y., Lee, W. E., 2000. Microstructural evolution in triaxial porcelain. Journal of the American Ceramic Society, 83, 3121-3127.
[34] Rambaldi, E., Carty, W. M., Tucci, A., Esposito, L., 2007. Using waste glass as a partial flux substitution and pyroplastic deformation of a porcelain stoneware tile body. Ceramics International, 33, 727-733.

[35] Raimondo, M., Zanelli, C., Guarini, G., Dondi, M., Fabbroni, R., Cortesi, T., 2009. Process of pyroplastic shaping for specialpurpose porcelain stoneware tiles.Ceramics International, $35,1975-1984$.

[36] Suvaci, E., Tamsu, N., 2010. The role of viscosity on microstructure development and stain resistance in porcelain stoneware tiles. Journal of the European Ceramic Society, 30, 3071-3077.

[37] Tunçel, D. Y., Kara, M. K., Özel, E., 2011. Effect of the chemical composition on the pyroplastic deformation of sanitaryware porcelain body. IOP Conference Series: Materials Science and Engineering, 18, 22, 222025.

[38] Aydin, T., Kara, A., 2014. Effect of spodumene addition on pyroplastic deformation of porcelain stoneware. Journal of Ceramic Processing Research, 15, 486-491.

[39] dos Santos Conserva, L. R., Melchiades, F. G., Nastri, S., Boschi, A. O., Dondi, M., Guarini, G., Raimondo M., Zanelli, C., 2017. Pyroplastic deformation of porcelain stoneware tiles: Wet vs. dry processing. Journal of the European Ceramic Society, 37, 333-342.

[40] Paganelli, M., Sighinolfi, D., 2009. Effect of quartz particle size on porcelain stoneware sintering by means of optical dilatometry. CFI Ceramic Forum International, 86, E41-E45.

[41] Tucci, A., Esposito, L., Malmusi, L., Rambaldi, E., 2007. New body mixes for porcelain stoneware tiles with improved mechanical characteristics. Journal of the European Ceramic Society, 27(2), 1875-1881.

[42] Gualtieri, A. F., 2009. Development of Low-Firing B-Fluxed Stoneware Tiles. Journal of the American Ceramic Society, 92, 2571-2577.

[43] Çigdemir, G., Kara, A., Kara, F., 2010. Effect of alkaline earth oxides on firing behaviour of porcelain stoneware. Industrial Ceramics, 30, 177-186.

[44] Melchiades, F. G., Santos, L. R., Boschi, A. O. Nastri S., 2012. Gres porcelánico esmaltado producido por vía seca: materias primas fundentes. Boletín de la Sociedad Española de Cerámica y Vídrio, 51, 133-138.

[45] Tamsu, N., Bayrak, A. V., Ozdemir, H., 2013. Effects of $\mathrm{Na}_{2} \mathrm{O} / \mathrm{K}_{2} \mathrm{O}$ Ratio on the Deformation Behaviour of the Floor Tile Bodies. Acta Physica Polonica, A123, 283-284.

[46] Magagnin, D., dos Santos, C. M. F., Wanderlind, A., Jiusti, J., De Noni, A., 2014. Effect of kaolinite, illite and talc on the processing properties and mullite content of porcelain stoneware tiles. Materials Science and Engineering, A618, 533-539.

[47] Adamo, I., Diella, V., Pavese, A., Vignola, P., Francescon, F., 2013. Na-feldspar (F) and kaolinite (K) system at high temperature: Resulting phase composition, micro-structural features and mullite-glass Gibbs energy of formation, as a function of $\mathrm{F} / \mathrm{K}$ ratio and kaolinite crystallinity. Journal of the European Ceramic Society, 33, 3387-3395.

[48]Becker, C. R., Carty, M., 1999. The role of flux choice in triaxial whiteware bodies. Ceramic Engineering Science Proceedings, 20(2), 43-50.

[49]Bernasconi, A., Marinoni, N., Pavese, A., Francescon, F., Young, K., 2014. Feldspar and firing cycle effects on the evolution of sanitary-ware vitreous body. Ceramics International, 40, 6389-6398. 
[50]Dana, K., Das, S.K.R., 2004. Effect of Na-feldspar and $\mathrm{K}$-feldspar on the whiteness and other properties of porcelain bodies. Industrial Ceramics, 24, 91-95.

[51]Das, S. K., Dana, K., 2003. Differences in densification behaviour of K-and Na-feldspar-containing porcelain bodies. Thermochimica Acta, 406, 199-206.

[52]Zanelli, C., Baldi, G., Dondi, M., Ercolani, G., Guarini, G., Raimondo, M., 2008. Glass-ceramic frits for porcelain stoneware bodies: Effects on sintering phase composition and technological properties. Ceramics International, 34, 455-465.

[53] Romero, M., Martín-Márquez, J., Rincón, J. M., 2006. Kinetic of mullite formation from a porcelain stoneware body for tiles production. Journal of the European Ceramic Society, $26,1647-1652$.

[54] Lee, W. E., Souza, G. P., McConville, C. J., Tarvornpanich, T., Iqbal, Y., 2008. Mullite formation in clays and clay derived vitreous ceramics. Journal of the European Ceramic Society, 28, 465-471.

[55] Martín-Márquez, J., Rincón, J. M., Romero, M., 2010. Mullite development on firing in porcelain stoneware bodies. Journal of the European Ceramic Society, 30, 1599-1607.

[56] Pérez, J. M., Rincón, J. M., Romero, M., 2010. Study of mullite formation in porcelain stoneware applying isoconversional and IKP methods. Ceramics International, 36, 2329-2335.

[57] Marinoni, N., Pagani, A., Adamo, I., Diella, V., Pavese, A., Francescon, F., 2011. Kinetic study of mullite growth in sanitary-ware production by in situ HT-XRPD. The influence of the filler/flux ratio. Journal of the European Ceramic Society, 31, 273-280.

[58] Marinoni, N., D’Alessio, D., Diella, V., Pavese, A., Francescon, F., 2013. Effects of soda-lime-silica waste glass on mullite formation kinetics and microstructures development in vitreous ceramics. Journal of Environmental Management, 124, 100-107.

[59]Pérez, J. M., Rincón, J. M., Romero, M., 2012. Effect of moulding pressure on microstructure and technological properties of porcelain stoneware. Ceramics International, 38(1), 317-325.

[60]Kirchhoff, G., Pompe, W., Bahr, H. A., 1982. Structure dependence of thermally induced microcracking in porcelain studied by acoustic emission. Journal of Materials Science, 17(10), 2809-2816.

[61]De Noni, A., Hotza, D., Soler, V. C., Vilches, E. S., 2008. Analysis of the development of microscopic residual stresses on quartz particles in porcelain tile. Journal of the European Ceramic Society, 28(14), 2629-2637.

[62] De Noni, A., Hotza, D., Soler, V. C., Vilches, E. S., 2009. Effect of quartz particle size on the mechanical behavior of porcelain tile subjected to different cooling rates. Journal of the European Ceramic Society, 29(6), 1039-1046.

[63] Gilabert, F. A., Dal Bo, M., Cantavella, V., Sánchez, E., 2012. Fracture patterns of quartz particles in glass feldspar matrix. Materials Letters, 72, 148-152.

[64] Reinosa, J. J., del Campo, A., Fernández, J. F., 2015. Indirect measurement of stress distribution in quartz particles embedded in a glass matrix by using confocal Raman microscopy. Ceramics International, 41(10), 13598-13606.

[65]Dal Bó, M., Cantavella, V., Sánchez, E., Hotza, D., Boschi, A., 2012. Mechanical modelling of rapid cooling in porcelain tile-type systems. Boletín de la Sociedad Española de Cerámica y Vídrio, 51(2), 95-102.

[66] Dal Bó, M., Cantavella, V., Sanchéz, E., Gilabert, F. A., Boschi, A. O., Hotza, D., 2017. An estimate of quartz content and particle size in porcelain tiles from Young's modulus measurements. Ceramics International, 43, 2233-2238.

[67] Martín-Márquez, J., Rincón, J. M., \& Romero, M., 2008. Effect of firing temperature on sintering of porcelain stoneware tiles. Ceramics International, 34(8), 1867-1873.

[68] De Noni, A., Hotza, D., Soler, V. C., \& Vilches, E. S., 2011. Influence of composition on mechanical behaviour of porcelain tile. Part III: Effect of the cooling rate of the firing cycle. Materials Science and Engineering A 528, 3330-3336.

[69] Lassinantti Gualtieri, M., Romagnoli, M., \& Gualtieri, A. F., 2011. Influence of body composition on the technological properties and mineralogy of stoneware: A DOE and mineralogical-microstructural study. Journal of the European Ceramic Society, 31, 673-685.

[70] Carty, W.M., Senapati, U., 1998. Porcelain - Raw materials, processing, phase evolution, and mechanical behavior. Journal of the American Ceramic Society, 81, 3-20.

[71]Zanelli, C., Dondi, M., Guarini, G., Raimondo, M., Roncarati, I., 2004. Influence of strengthening components on industrial mixture of porcelain stoneware tiles. Key Engineering Materials, 264-268, 1491-1494.

[72]De Noni, A., Hotza, D., Soler, V. C., Vilches, E. S., 2010. Influence of composition on mechanical behaviour of porcelain tile. Part II: Mechanical properties and microscopic residual stress. Materials Science and Engineering A, 527, 1736-1743.

[73]Lee, W. E., Iqbal, Y., 2001. Influence of mixing on mullite formation in porcelain. Journal of the European Ceramic Society, 21(14), 2583-2586.

[74]Prado, M. O., Zanotto, E. D., \& Müller, R., 2001. Model for sintering polydispersed glass particles. Journal of NonCrystalline Solids, 279, 169-178.

[75] Perugini, D., De Campos, C. P., Petrelli, M., Dingwell, D. B., 2015. Concentration variance decay during magma mixing: a volcanic chronometer. Scientific Reports, 5, 14225.

[76]Dannert, C., Durschang, B., Raether, F., Becker, F., 2003. Optimization of sintering processes for porcelain using insitu measuring methods. Glass Science and Technology, 76, 71.

[77] Reijnen, P., 1996. Pore growth and elimination during sintering of silicate ceramics. CFI Ceramic forum international, 73(10), 594-598.

[78] Takahashi, H., Takeuchi, N., Ishida, S., \& Wakamatsu, M., 2000. Oxygen evolution and chemical state changes of iron during firing of red clay bodies at low oxygen pressure. Journal of the ceramic Society of Japan, 108(1260), 705-709.

[79] Dingwell, D. B., \& Virgo, D., 1987. The effect of oxidation state on the viscosity of melts in the system $\mathrm{Na}_{2} \mathrm{O}-\mathrm{FeO}-\mathrm{Fe}_{2} \mathrm{O}_{3}$ $\mathrm{SiO}_{2}$. Geochimica et Cosmochimica Acta, 51(2), 195-205. 